\title{
WEAK TYPE COMMUTATOR AND LIPSCHITZ ESTIMATES: RESOLUTION OF THE NAZAROV-PELLER CONJECTURE
}

\author{
M. CASPERS, D. POTAPOV, F. SUKOCHEV, D. ZANIN
}

\begin{abstract}
Let $\mathcal{M}$ be a semi-finite von Neumann algebra and let $f: \mathbb{R} \rightarrow \mathbb{C}$ be a Lipschitz function. If $A, B \in \mathcal{M}$ are self-adjoint operators such that $[A, B] \in L_{1}(\mathcal{M})$, then

$$
\|[f(A), B]\|_{1, \infty} \leq c_{a b s}\left\|f^{\prime}\right\|_{\infty}\|[A, B]\|_{1}
$$

where $c_{a b s}$ is an absolute constant independent of $f, \mathcal{M}$ and $A, B$ and $\|\cdot\|_{1, \infty}$ denotes the weak $L_{1}$-norm. If $X, Y \in \mathcal{M}$ are self-adjoint operators such that $X-Y \in L_{1}(\mathcal{M})$, then

$$
\|f(X)-f(Y)\|_{1, \infty} \leq c_{a b s}\left\|f^{\prime}\right\|_{\infty}\|X-Y\|_{1} .
$$

This result resolves a conjecture raised by F. Nazarov and V. Peller implying a couple of existing results in perturbation theory.
\end{abstract}

\section{INTRODUCTION}

Let $L_{p}(H)$ be the Schatten-von Neumann ideal of $B(H)$. It consists of all compact operators for which its sequence of singular values lies in $\ell_{p}$. Let $F_{p}$ be the class of functions $f: \mathbb{R} \rightarrow \mathbb{C}$ such that

$$
f(B)-f(C) \in L_{p}(H),
$$

for all self-adjoint $B, C$ such that $B-C \in L_{p}(H)$ and set

$$
\|f\|_{F_{p}}=\sup _{B \neq C} \frac{\|f(B)-f(C)\|_{p}}{\|B-C\|_{p}} .
$$

It was conjectured by M.G. Krein [17] that whenever the derivative $f^{\prime} \in L_{\infty}(\mathbb{R})$ we have $f \in F_{1}$. This conjecture does not hold as was shown by Y.B. Farforovskaya in [11. Also it was shown that the analogue of Krein's problem fails in the case $p=\infty$ (see [9, 10]). In fact already for the absolute value function it was found by T. Kato that Krein's problem has a negative answer [15]; and similarly in the case $p=1$ by E.B. Davies [5].

A positive result in this direction was first obtained by M. Birman and M. Solomyak [1, Theorem 10] who proved that $C^{1+\epsilon} \subseteq F_{1}$ for every $\epsilon>0$, and later improved by V. Peller [22] who showed that $B_{\infty 1}^{1} \subseteq F_{1}$. Here $B_{p q}^{s}$ is the class of Besov spaces for which we refer to 12. The Krein problem for the case $1<p<\infty, p \neq 2$ remained open until 24. In 24 it was shown by the second and third named author that $F_{p}$ consists exactly of all Lipschitz functions. Moreover in [3] a quantitative estimate for $\|f\|_{F_{p}}$ was found, namely $\|f\|_{F_{p}} \simeq p^{2} /(p-1)$. Earlier the same problem had been considered by M. de la Salle (unpublished, see

Date: June 3, 2015, MSC2000: 47B10, 47L20, 47A30, Acknowledgement: The first author is supported by the grant SFB 878. The work of other coauthors is supported by the ARC. 
[27]) who was able to show already that $\|f\|_{F_{p}} \leq c_{a b s} \cdot p^{4} /(p-1)^{2}$ with an absolute constant $c_{a b s}$, which is more optimal than [24].

Other results concerning this problem have been obtained in [8] and [16] and in the context of this paper we also mention [26] in which weak estimates for martingale inequalities were obtained.

Using interpolation, the above results would follow from a weak type Lipschitz estimate between $L_{1}$ and the weak- $L_{1}$ space $L_{1, \infty}$. The estimate was conjectured in a paper of F. Nazarov and V. Peller [19], and has remained the major open question in the study of Lipschitz properties of operator valued functions. Denote $L_{1, \infty}(H)$ for the weak $L_{1}$-space consisting of all compact operators $A$ whose sequence $\{\mu(k, A)\}_{k \geq 0}$ of singular values satisfies $\mu(k, A)=O\left(\frac{1}{k+1}\right)$.

Conjecture 1.1. Let $f: \mathbb{R} \rightarrow \mathbb{C}$ be Lipschitz. Whenever $A, B \in B(H)$ are selfadjoint operators such that $A-B \in L_{1}(H)$, we have that $f(A)-f(B) \in L_{1, \infty}(H)$ and

$$
\|f(A)-f(B)\|_{1, \infty} \leq c_{a b s}\left\|f^{\prime}\right\|_{\infty}\|A-B\|_{1}
$$

for some absolute constant $c_{a b s}$.

Nazarov and Peller [19] gave an affirmative answer under the assumption that the rank of $A-B$ equals 1 . Since $\|\cdot\|_{1, \infty}$ is a quasi-norm and not a norm for $L_{1, \infty}(H)$ it is impossible to extend their result for when $A-B$ is a general trace class operator.

Another positive result to the conjecture was found by the current authors in [4] in the special case when $f$ is the absolute value map. The proof relies on the observation that the Schur multiplier of divided differences

$$
\left(\frac{f(\lambda)-f(\mu)}{\lambda-\mu}\right)_{\lambda \neq \mu}
$$

can be written as a finite sum of compositions of a positive definite Schur multiplier and a triangular truncation operator. For general Lipschitz functions there is no reason that the latter fact should be true which renders the technique of [4] inapplicable.

The main result of this paper is a proof of Conjecture 1.1. The importance of this result lies in the fact that this gives the sharpest possible estimate for perturbations and commutators. In particular it retrieves $\|f\|_{F_{p}} \simeq p^{2} /(p-1)[3$ and the Nazarov-Peller result [19]. A key ingredient in our proof is the connection with non-commutative Calderón-Zygmund theory and in particular J. Parcet's extension of the classical Calderón-Zygmund theorem (see Theorem 2.1] and 21]).

In the text we prove a somewhat stronger result in the terms of double operator integrals (see next section for definition), of which Conjecture 1.1 is a corollary.

Theorem 1.2. If $A$ is a self-adjoint operator affiliated with a semifinite von Neumann algebra $\mathcal{M}$, and if $f: \mathbb{R} \rightarrow \mathbb{C}$ is Lipschitz then

$$
\left\|T_{f^{[1]}}^{A, A}(V)\right\|_{1, \infty} \leq c_{a b s}\left\|f^{\prime}\right\|_{\infty}\|V\|_{1}, \quad V \in\left(L_{1} \cap L_{2}\right)(\mathcal{M}) .
$$

Commutator estimate follows from the observation that the double operator integral $T_{f^{[1]}}^{A, A}([A, B])$ equals $[f(A), B]$. As explained in the proof of Theorem 5.3 . Lipschitz estimates follow from commutator ones. 
Acknowledgements. Authors thank Javier Parcet for a detailed explanation of 21].

\section{Preliminaries}

2.1. General notation. Let $\mathcal{M}$ be a semifinite von Neumann algebra equipped with a faithful normal semifinite trace $\tau$. In this paper, we always presume that $\mathcal{M}$ is represented on a separable Hilbert space.

A (closed and densely defined) operator $x$ affiliated to $\mathcal{M}$ is called $\tau$-measurable if $\tau\left(E_{|x|}(s, \infty)\right)<\infty$ for sufficiently large $s$. We denote the set of all $\tau$-measurable operators by $S(\mathcal{M}, \tau)$. For every $x \in S(\mathcal{M}, \tau)$, we define its singular value function $\mu(A)$ by setting

$$
\mu(t, x)=\inf \left\{\|x(1-p)\|_{\infty}: \quad \tau(p) \leq t\right\} .
$$

Equivalently, for positive operator $x \in S(\mathcal{M}, \tau)$, we have

$$
n_{x}(s)=\tau\left(E_{x}(s, \infty)\right), \quad \mu(t, x)=\inf \left\{s: n_{x}(s)<t\right\} .
$$

We have (see e.g. [18, Corollary 2.3.16])

$$
\mu(t+s, x+y) \leq \mu(t, x)+\mu(s, y), \quad t, s>0 .
$$

2.2. Non-commutative spaces. For $1 \leq p<\infty$ we set,

$$
L_{p}(\mathcal{M})=\left\{x \in S(\mathcal{M}, \tau): \tau\left(|x|^{p}\right)<\infty\right\}, \quad\|x\|_{p}=\left(\tau\left(|x|^{p}\right)\right)^{\frac{1}{p}} .
$$

The Banach spaces $\left(L_{p}(\mathcal{M}),\|\cdot\|_{p}\right), 1 \leq p<\infty$ are separable.

Define the space $L_{1, \infty}(\mathcal{M})$ by setting

$$
L_{1, \infty}(\mathcal{M})=\left\{x \in S(\mathcal{M}, \tau): \sup _{t>0} t \mu(t, x)<\infty\right\} .
$$

We equip $L_{1, \infty}(\mathcal{M})$ with the functional $\|\cdot\|_{1, \infty}$ defined by the formula

$$
\|x\|_{1, \infty}=\sup _{t>0} t \mu(t, x), \quad x \in L_{1, \infty}(\mathcal{M}) .
$$

It follows from (2.1) that

$$
\begin{gathered}
\|x+y\|_{1, \infty}=\sup _{t>0} t \mu(t, x+y) \leq \sup _{t>0} t\left(\mu\left(\frac{t}{2}, x\right)+\mu\left(\frac{t}{2}, y\right)\right) \leq \\
\leq \sup _{t>0} t \mu\left(\frac{t}{2}, x\right)+\sup _{t>0} t \mu\left(\frac{t}{2}, y\right)=2\|x\|_{1, \infty}+2\|y\|_{1, \infty} .
\end{gathered}
$$

In particular, $\|\cdot\|_{1, \infty}$ is a quasi-norm. The quasi-normed space $\left(L_{1, \infty}(\mathcal{M}),\|\cdot\|_{1, \infty}\right)$ is, in fact, quasi-Banach (see e.g. [14, Section 7] or [30). In view of our main result it is important to emphasize that the quasi-norm $\|\cdot\|_{1, \infty}$ is not equivalent to any norm on $L_{1, \infty}(\mathcal{M})$ (see e.g [14, Theorem 7.6]).

2.3. Weak type inequalities for Calderón-Zygmund operators. Parcet 21$]$ proved a noncommutative extension of Calderón-Zygmund theory.

Let $K$ be a tempered distribution which we refer to as the convolution kernel. We let $W_{K}$ be the associated Calderón-Zygmund operator, formally given by $f \mapsto K * f$. In what follows, we only consider tempered distributions having local values (that is, which can be identified with measurable functions $K: \mathbb{R}^{d} \rightarrow \mathbb{C}$ ).

Let $\mathcal{M}$ be a semi-finite von Neumann algebra with normal, semi-finite, faithful trace $\tau$. The operator $1 \otimes W_{K}$ can, under suitable conditions, be defined as noncommutative Calderón-Zygmund operators by letting them act on the second tensor leg 
of $L_{1}(\mathcal{M}) \widehat{\otimes} L_{1}\left(\mathbb{R}^{d}\right)$. The following theorem in particular gives a sufficient condition for such an operator to act from $L_{1}$ to $L_{1, \infty}$.

Theorem 2.1 (21]). Let $K: \mathbb{R}^{d} \backslash\{0\} \rightarrow \mathbb{C}$ be a kernel satisfying the condition $₫$

$$
|K|(t) \leq \frac{\text { const }}{|t|^{d}}, \quad|\nabla K|(t) \leq \frac{\text { const }}{|t|^{d+1}} .
$$

Let $\mathcal{M}$ be a semi-finite von Neumann algebra. If $W_{K} \in B\left(L_{2}\left(\mathbb{R}^{d}\right)\right)$, then the operator $1 \otimes W_{K}$ defines a bounded map from $L_{1}\left(\mathcal{M} \otimes L_{\infty}\left(\mathbb{R}^{d}\right)\right)$ to $L_{1, \infty}\left(\mathcal{M} \otimes L_{\infty}\left(\mathbb{R}^{d}\right)\right)$.

2.4. Double operator integrals. Let $A=A^{*}$ be an operator affiliated with $\mathcal{M}$. Symbolically, a double operator integral is defined by the formula

$$
T_{\xi}^{A, A}(V)=\int_{\mathbb{R}^{2}} \xi(\lambda, \mu) d E_{A}(\lambda) V E_{A}(\mu), \quad V \in L_{2}(\mathcal{M}) .
$$

In the subsequent paragraph, we provide a rigorous definition of the double operator integral.

Consider projection valued measures on $\mathbb{R}$ acting on the Hilbert space $L_{2}(\mathcal{M})$ by the formulae $x \rightarrow E_{A}(\mathcal{B}) x$ and $x \rightarrow x E_{A}(\mathcal{B})$. These spectral measures commute and, hence (see Theorem V.2.6 in [2]), there exists a countably additive (in the strong operator topology) projection-valued measure $\nu$ on $\mathbb{R}^{2}$ acting on the Hilbert space $L_{2}(\mathcal{M})$ by the formula

$$
\nu\left(\mathcal{B}_{1} \otimes \mathcal{B}_{2}\right): x \rightarrow E_{A}\left(\mathcal{B}_{1}\right) x E_{A}\left(\mathcal{B}_{2}\right), \quad x \in L_{2}(\mathcal{M}) .
$$

Integrating a bounded Borel function $\xi$ on $\mathbb{R}^{2}$ with respect to the measure $\nu$ produces a bounded operator acting on the Hilbert space $L_{2}(\mathcal{M})$. In what follows, we denote the latter operator by $T_{\xi}^{A, A}$ (see also [20, Remark 3.1]).

In the special case when $A$ is bounded and $\operatorname{spec}(A) \subset \mathbb{Z}$, we have

$$
T_{\xi}^{A, A}(V)=\sum_{k, l \in \mathbb{Z}} \xi(k, l) E_{A}(\{k\}) V E_{A}(\{l\}) .
$$

We are mostly interested in the case $\xi=f^{[1]}$ for a Lipschitz function $f$. Here,

$$
f^{[1]}(\lambda, \mu)=\left\{\begin{array}{l}
\frac{f(\lambda)-f(\mu)}{\lambda-\mu}, \quad \lambda \neq \mu \\
0, \quad \lambda=\mu .
\end{array}\right.
$$

\section{Approximate intertwining Properties of Fourier multipliers}

We prove intertwining properties of Fourier multipliers partly inspired by K. de Leeuw's proof of his restriction theorem for $L^{p}$-multipliers [6, Section 2].

In what follows,

$$
G_{l}(s)=\frac{1}{l \sqrt{\pi}} e^{-\left(\frac{s}{l}\right)^{2}}, \quad s \in \mathbb{R}, \quad l>0 .
$$

That is, $G_{l}$ is a probability density function for certain Gaussian random variable. The notation $G_{l}^{\otimes d}$ stands for the function from $L_{1}\left(\mathbb{R}^{d}\right)$ given by the tensor product of $G_{l}$ with itself repeated $d$ times.

Lemma 3.1. For every $f \in L_{1}(\mathbb{R})$ with $\int_{-\infty}^{\infty} f(s) d s=0$, we have $f * G_{l} \rightarrow 0$ in $L_{1}(\mathbb{R})$ as $l \rightarrow \infty$.

\footnotetext{
${ }^{1}$ Here, $\nabla$ denotes the gradient $\left(\frac{1}{i} \frac{\partial}{\partial x_{1}}, \cdots, \frac{1}{i} \frac{\partial}{\partial x_{d}}\right)$, which is understood as unbounded selfadjoint operator on $L_{2}\left(\mathbb{R}^{d}\right)$.
} 
Proof. Suppose first that $f$ is a step function of the form $f=\sum_{k=1}^{m} \alpha_{k} \chi_{I_{k}}, m \geq 1$ where $I_{k}=\left[a_{k}, b_{k}\right], 1 \leq k \leq m$ are disjoint intervals and $\sum_{k=1}^{m} \alpha_{k} m\left(I_{k}\right)=0$.

We have

$$
\begin{gathered}
\left(f * G_{l}\right)(t)=\sum_{k=1}^{m} \alpha_{k} \int_{a_{k}}^{b_{k}} G_{l}(t-s) d s=\sum_{k=1}^{m} \alpha_{k} \int_{t-b_{k}}^{t-a_{k}} G_{l}(u) d u= \\
=\sum_{k=1}^{m} \alpha_{k} \int_{\frac{t-b_{k}}{l}}^{\frac{t-a_{k}}{l}} G_{1}(s) d s=\sum_{k=1}^{m} \alpha_{k}\left(F\left(\frac{t-a_{k}}{l}\right)-F\left(\frac{t-b_{k}}{l}\right)\right),
\end{gathered}
$$

where $F(t)=\int_{-\infty}^{t} G_{1}(s) d s$. To prove the assertion for our $f$, it suffices to show that

$$
l \int_{-\infty}^{\infty}\left|\sum_{k=1}^{m} \alpha_{k}\left(F\left(t-\frac{a_{k}}{l}\right)-F\left(t-\frac{b_{k}}{l}\right)\right)\right| d t \rightarrow 0
$$

Clearly,

$$
\left|F\left(t-\frac{a_{k}}{l}\right)-F(t)+\frac{a_{k}}{l} F^{\prime}(t)\right| \leq \frac{a_{k}^{2}}{2 l^{2}} \max _{s \in\left[t-\frac{a_{k}}{l}, t\right]}\left|F^{\prime \prime}(s)\right| .
$$

If $l>\max _{1 \leq k \leq m}\left|a_{k}\right|$ and $l>\max _{1 \leq k \leq m}\left|b_{k}\right|$, then

$$
\left|\sum_{k=1}^{m} \alpha_{k}\left(F\left(t-\frac{a_{k}}{l}\right)-F\left(t-\frac{b_{k}}{l}\right)\right)\right| \leq \frac{1}{2 l^{2}}\left(\sum_{k=1}^{m}\left|\alpha_{k}\right|\left(a_{k}^{2}+b_{k}^{2}\right)\right) \max _{s \in[t-1, t+1]}\left|F^{\prime \prime}(s)\right| .
$$

This proves the assertion for $f$ as above.

To prove the assertion in general, fix $f_{m}$ as above (i.e., mean zero step functions) such that $f_{m} \rightarrow f$ in $L_{1}(\mathbb{R})$. Since $\left\|G_{l}\right\|_{1}=1$, it follows from Young's inequality that

$$
\left\|f * G_{l}\right\|_{1} \leq\left\|\left(f-f_{m}\right) * G_{l}\right\|_{1}+\left\|f_{m} * G_{l}\right\|_{1} \leq\left\|f-f_{m}\right\|_{1}+\left\|f_{m} * G_{l}\right\|_{1} .
$$

Therefore,

$$
\limsup _{l \rightarrow \infty}\left\|f * G_{l}\right\|_{1} \leq\left\|f-f_{m}\right\|_{1} .
$$

Passing $m \rightarrow \infty$, we conclude the proof.

By Fubini Theorem, linear span of elementary tensors

$$
\left(f_{1} \otimes \cdots \otimes f_{d}\right):\left(t_{1}, \cdots, t_{d}\right) \rightarrow f_{1}\left(t_{1}\right) \cdots f_{d}\left(t_{d}\right), \quad f_{1}, \cdots, f_{d} \in L_{1}(\mathbb{R})
$$

is dense in $L_{1}\left(\mathbb{R}^{d}\right)$.

Lemma 3.2. For every $f \in L_{1}\left(\mathbb{R}^{d}\right)$ with $\int_{\mathbb{R}^{d}} f(s) d s=0$, we have $f * G_{l}^{\otimes d} \rightarrow 0$ in $L_{1}\left(\mathbb{R}^{d}\right)$ as $l \rightarrow \infty$.

Proof. Suppose first that $f$ is a linear combination of elementary tensors. That is,

$$
f=\sum_{k=1}^{m} \bigotimes_{j=1}^{d} f_{j k}, \quad f_{j k} \in L_{1}(\mathbb{R}) .
$$

Firstly, we consider the case when for every $k, 1 \leq k \leq m$ there exists $j, 1 \leq j \leq d$ such that $\int_{\mathbb{R}} f_{j k}(s)=0$. In this case, by Lemma 3.1 we have that

$$
\left\|f * G_{l}^{\otimes d}\right\|_{1} \leq \sum_{k=1}^{m} \prod_{j=1}^{d}\left\|f_{j k} * G_{l}\right\|_{1} \rightarrow 0 .
$$


Now, we show that the case of $f$ given by (3.1) satisfying

$$
\sum_{k=1}^{m} \prod_{j=1}^{d} \int_{\mathbb{R}} f_{j k}(s) d s=0 .
$$

can be reduced to the just considered case when for every $k, 1 \leq k \leq m$ there exists $j, 1 \leq j \leq d$ such that $\int_{\mathbb{R}} f_{j k}(s)=0$. To this end, for every subset $\mathscr{A} \subset\{1, \cdots, d\}$, we set

$$
f_{j, k, \mathscr{A}}=\left\{\begin{array}{l}
f_{j k}-\left(\int_{\mathbb{R}} f_{j k}(s) d s\right) \chi_{(0,1)}, \quad j \in \mathscr{A} \\
\left(\int_{\mathbb{R}} f_{j k}(s) d s\right) \chi_{(0,1)}, \quad j \notin \mathscr{A} .
\end{array}\right.
$$

By the linearity, we can rewrite (3.1) as

$$
f=\sum_{k=1}^{m} \sum_{\mathscr{A} \subset\{1, \cdots, d\}} \bigotimes_{j=1}^{d} f_{j, k, \mathscr{A}}
$$

Observing now that

$$
\sum_{k=1}^{m} \bigotimes_{j=1}^{d} f_{j, k, \varnothing}=\left(\sum_{k=1}^{m} \prod_{j=1}^{d} \int_{\mathbb{R}} f_{j k}(s) d s\right) \chi_{(0,1)}^{\otimes d}
$$

and appealing to (3.2), we arrive at

$$
f=\sum_{k=1}^{m} \sum_{\varnothing \neq \mathscr{A} \subset\{1, \cdots, d\}} \bigotimes_{j=1}^{d} f_{j, k, \mathscr{A}} .
$$

Note that $f_{j, k, \mathscr{A}}$ is mean zero for $j \in \mathscr{A}$. Using representation (3.3) instead of (3.1) for $f$, we may assume without loss of generality that for every $k, 1 \leq k \leq m$ there exists $j, 1 \leq j \leq d$ such that $\int_{\mathbb{R}} f_{j k}(s)=0$. This completes the proof of the lemma in the special case when $f$ is given by (3.1) and satisfies (3.2).

To prove the general case, fix $f \in L_{1}\left(\mathbb{R}^{d}\right)$ with $\int_{\mathbb{R}^{d}} f(s) d s=0$, and select a sequence $\left\{f_{m}\right\}_{m=1}^{\infty}$ of mean zero sums of elementary tensors such that $f_{m} \rightarrow f$ in $L_{1}\left(\mathbb{R}^{d}\right)$ as $m \rightarrow \infty$. Since $\left\|G_{l}^{\otimes d}\right\|_{1}=1, l \geq 1$ it follows from Young inequality that

$$
\left\|f * G_{l}^{\otimes d}\right\|_{1} \leq\left\|\left(f-f_{m}\right) * G_{l}^{\otimes d}\right\|_{1}+\left\|f_{m} * G_{l}^{\otimes d}\right\|_{1} \leq\left\|f-f_{m}\right\|_{1}+\left\|f_{m} * G_{l}^{\otimes d}\right\|_{1} .
$$

Therefore,

$$
\limsup _{l \rightarrow \infty}\left\|f * G_{l}^{\otimes d}\right\|_{1} \leq\left\|f-f_{m}\right\|_{1} .
$$

Passing $m \rightarrow \infty$, we conclude the proof.

In what follows,

$$
e_{k}(t):=e^{i\langle k, t\rangle}, \quad k, t \in \mathbb{R}^{d}
$$

and $\mathcal{F}$ stands for the Fourier transform.

Lemma 3.3. If $g \in L_{\infty}\left(\mathbb{R}^{d}\right)$ is such that $\mathcal{F}(g) \in L_{1}\left(\mathbb{R}^{d}\right)$, then for every $k \in \mathbb{R}^{d}$ we have

$$
(g(\nabla))\left(G_{l}^{\otimes d} e_{k}\right)-g(k) G_{l}^{\otimes d} e_{k} \rightarrow 0
$$

in $L_{1}\left(\mathbb{R}^{d}\right)$ as $l \rightarrow \infty$. 
Proof. Fix $k \in \mathbb{R}^{d}$. Set $h_{1}(t):=g(k) e^{-|t-k|^{2}}$ and $h_{0}(t):=g(t)-h_{1}(t), t \in \mathbb{R}^{d}$. Observe that, for every $t \in \mathbb{R}^{d}$, we have

$$
\mathcal{F}\left(G_{l}^{\otimes d}\right)(t)=\pi^{-d / 2} e^{-l^{2}|t|^{2}} .
$$

Since $h_{1}(\nabla)$ on the Fourier side is a multiplier on $h_{1}$, it follows that, for every $t \in \mathbb{R}^{d}$,

$$
\mathcal{F}\left(G_{l}^{\otimes d} e_{k}\right)(t)=\pi^{-d / 2} e^{-l^{2}|t-k|^{2}}, \quad\left(\mathcal{F}\left(\left(h_{1}(\nabla)\right)\left(G_{l}^{\otimes d} e_{k}\right)\right)\right)(t)=g(k) \pi^{-d / 2} e^{-\left(l^{2}+1\right)|t-k|^{2}} .
$$

Applying the inverse Fourier transform to the second equality, we arrive at

$$
\left(h_{1}(\nabla)\right)\left(G_{l}^{\otimes d} e_{k}\right)=g(k) G_{\left(l^{2}+1\right)^{1 / 2}}^{\otimes d} e_{k} .
$$

A direct computation yields $G_{\left(l^{2}+1\right)^{1 / 2}}^{\otimes d}-G_{l}^{\otimes d} \rightarrow 0$ in $L_{1}\left(\mathbb{R}^{d}\right)$ as $l \rightarrow \infty$. We conclude that

$$
\left(h_{1}(\nabla)\right)\left(G_{l}^{\otimes d} e_{k}\right)-g(k) G_{l}^{\otimes d} e_{k} \rightarrow 0
$$

in $L_{1}\left(\mathbb{R}^{d}\right)$ as $l \rightarrow \infty$. It, therefore, suffices to show that

$$
\left(h_{0}(\nabla)\right)\left(G_{l}^{\otimes d} e_{k}\right) \rightarrow 0
$$

in $L_{1}\left(\mathbb{R}^{d}\right)$ as $l \rightarrow \infty$. Define the function $f \in L_{1}\left(\mathbb{R}^{d}\right)$ by setting $f(t)=e^{i\langle k, t\rangle}\left(\mathcal{F} h_{0}\right)(t)$, $t \in \mathbb{R}^{d}$. We rewrite the latter equation as $f * G_{l}^{\otimes d} \rightarrow 0$ as $l \rightarrow \infty$. Note that

$$
\int_{\mathbb{R}^{d}} f(s) d s=\int_{\mathbb{R}^{d}} e^{i\langle k, s\rangle}\left(\mathcal{F} h_{0}\right)(s) d s=h_{0}(k)=0 .
$$

The assertion follows now from the Lemma 3.2

\section{Proof of Theorem 1.2 in the special Case}

For $s>0$, the dilation operator $\sigma_{s}$ acts on the space of Lebesgue measurable functions on $\mathbb{R}$, by the formula $\left(\sigma_{s} x\right)(t)=x(t / s)$.

Lemma 4.1. Let $x, y$ be measurable and $\theta$ be integrable functions on $\mathbb{R}$. Let $z(t):=$ $t^{-1}, t>0, z(t)=0, t<0$, and let $u>0$. For Lebesgue measurable functions $x \otimes y$ and $\theta \otimes z$ on $\mathbb{R}^{2}$, we have

$$
\mu\left(\sigma_{u}(x) \otimes y\right)=\sigma_{u} \mu(x \otimes y), \quad \mu(t, \theta \otimes z)=\|\theta\|_{1} t^{-1}, \quad t>0 .
$$

Proof. Denoting Lebesgue measure on $\mathbb{R}^{2}$ by $m$, we have for every $t>0$

$$
\begin{aligned}
m\left(\left\{\sigma_{u}(x) \otimes y>t\right\}\right) & =m\left(\left\{\left(s_{1}, s_{2}\right): x\left(\frac{s_{1}}{u}\right) y\left(s_{2}\right)>t\right\}\right) \\
& =u m\left(\left\{\left(s_{1}, s_{2}\right): x\left(s_{1}\right) y\left(s_{2}\right)>t\right\}\right) \\
& =u m(\{x \otimes y>t\}) .
\end{aligned}
$$

This proves the first assertion.

Firstly, we prove the second assertion for simple function $x \in L_{1}(\mathbb{R})$. If $x=$ $\sum_{k} a_{k} \chi_{B_{k}}$ with $B_{k}$ being pairwise disjoint sets, then 2

$$
\mu(x \otimes z)=\mu\left(\bigoplus_{k}\left(a_{k} \chi_{B_{k}} \otimes z\right)\right)=\mu\left(\bigoplus_{k} \mu\left(\left(a_{k} \chi_{B_{k}} \otimes z\right)\right) .\right.
$$

\footnotetext{
${ }^{2}$ The notation $\bigoplus_{k} x_{k}$ stands for disjoint sum of the functions $x_{k}$, that is $\sum_{k} z_{k}$, where functions $z_{k}$ have pairwise disjoint support and $\mu\left(z_{k}\right)=\mu\left(x_{k}\right)$. We refer the reader to the Definition 2.4.3 in [18 and subsequent comments.
} 
If $B$ is a set of finite measure, then there exists a measure preserving bijection from $B$ to $(0, m(B))$ (see [13]). Therefore, we have

$$
\mu\left(\chi_{B} \otimes z\right)=\mu\left(\chi_{(0, m(B))} \otimes z\right)=m(B) z .
$$

Thus,

$$
\mu(x \otimes z)=\mu\left(\bigoplus_{k}\left|a_{k}\right| m\left(B_{k}\right) z\right)=\left(\sum_{k} a_{k} m\left(B_{k}\right)\right) z .
$$

The second assertion follows now by approximation.

Lemma 4.2. For every $X \in L_{1, \infty}(\mathcal{M})$ and every $l>0$, we have

$$
e^{-d} \pi^{-\frac{d}{2}}\|X\|_{1, \infty} \leq\left\|X \otimes G_{l}^{\otimes d}\right\|_{1, \infty} \leq\|X\|_{1, \infty} .
$$

Proof. For every operator $A \in S(\mathcal{M}, \tau)$ and for every function $g \in L_{\infty}(0, \infty)$, we have

$$
\mu(A \otimes g)=\mu(|A| \otimes g)=\mu(\mu(A) \otimes g)=\mu(\mu(A) \otimes \mu(g)) .
$$

Let $z$ be as in Lemma 4.1. It follows from the definition $\|\cdot\|_{1, \infty}$ that $\mu(X) \leq$ $\|X\|_{1, \infty} z$ and, therefore,

$$
\mu\left(X \otimes G_{l}^{\otimes d}\right) \stackrel{\text { 4.1) }}{=} \mu\left(\mu(X) \otimes G_{l}^{\otimes d}\right) \leq\|X\|_{1, \infty} \mu\left(z \otimes G_{l}^{\otimes d}\right) \stackrel{L{ }^{4.1}}{=}\|X\|_{1, \infty} \mu(z) .
$$

This proves the right hand side inequality.

On the other hand, $\mu\left(G_{l}\right)=l^{-1} \sigma_{l} \mu(G)$. By Lemma 4.1 we have $\mu\left(G_{l}^{\otimes d}\right)=$ $l^{-d} \sigma_{l^{d}} \mu\left(G_{1}^{\otimes d}\right)$. Thus,

$$
\mu\left(X \otimes G_{l}^{\otimes d}\right) \stackrel{4.1]}{=} \mu\left(X \otimes l^{-d} \sigma_{l^{d}} \mu\left(G_{1}^{\otimes d}\right)\right) \stackrel{L 4.1}{=} l^{-d} \sigma_{l^{d}} \mu\left(X \otimes G_{1}^{\otimes d}\right) .
$$

Therefore, we have

$$
\left\|X \otimes G_{l}^{\otimes d}\right\|_{1, \infty}=\sup _{t>0} \frac{t}{l^{d}} \mu\left(\frac{t}{l^{d}}, X \otimes G_{1}^{\otimes d}\right)=\sup _{s>0} s \mu\left(s, X \otimes G_{1}^{\otimes d}\right)=\left\|X \otimes G_{1}^{\otimes d}\right\|_{1, \infty} .
$$

Clearly, $\mu\left(G_{1}\right) \geq \frac{1}{e \sqrt{\pi}} \chi_{(0,1)}$. It follows that

$$
\left\|X \otimes G_{1}^{\otimes d}\right\|_{1, \infty} \stackrel{\text { (4.1) }}{=}\left\|X \otimes \mu\left(G_{1}\right)^{\otimes d}\right\|_{1, \infty} \geq\left\|X \otimes\left(\frac{1}{e \sqrt{\pi}} \chi_{(0,1)}\right)^{\otimes d}\right\|_{1, \infty}=e^{-d} \pi^{-\frac{d}{2}}\|X\|_{1, \infty} .
$$

This proves the left hand side inequality.

The following lemma is ideologically similar to Theorem II.4.3 in [28].

Lemma 4.3. If $g$ is a smooth homogeneous function on $\mathbb{R}^{2} \backslash\{0\}$, then $\mathcal{F} g$ satisfies (possibly, after some $\delta$ distribution is subtracted) the conditions (2.2).

Proof. By assumption, $g$ is a smooth function on the circle $\{|z|=1\}$. Thus,

$$
g\left(e^{i \theta}\right)=\sum_{k \in \mathbb{Z}} \alpha_{k} e^{i k \theta},
$$

\footnotetext{
${ }^{3}$ Without loss of generality, $\mathcal{M}$ is atomless. Suppose first that $x \in \mathcal{M}$ is $\tau$-compact. By Theorem 2.3.11 in [18], there exists a trace preserving $*$-isomorphism $i: L_{\infty}(0, \infty) \rightarrow \mathcal{M}_{1}$ such that $i_{1}(\mu(A))=|A|$. Consider trace preserving isomorphism $i \otimes 1: L_{\infty}(0, \infty) \otimes L_{\infty}(0, \infty) \rightarrow$ $\mathcal{M} \otimes L_{\infty}(0, \infty)$. We have $i(\mu(A) \otimes g)=|A| \otimes g$. Since every trace preserving $*$-isomorphism preserves singular value function, the claim follows for $\tau$-compact operators. The general case follows by approximation.
} 
where Fourier coefficients decrease faster than every power. Therefore,

$$
g=\sum_{k \in \mathbb{Z}} \alpha_{k} g_{k}, \quad g_{k}(z)=\frac{z^{k}}{|z|^{k}}, \quad 0 \neq z \in \mathbb{C} .
$$

For every $k \neq 0$, we hav屯

$$
\left(\mathcal{F} g_{k}\right)(z)=\frac{|k|}{2 \pi i^{k}} \cdot \frac{g_{k}(z)}{|z|^{2}}, \quad 0 \neq z \in \mathbb{C} .
$$

Hence,

$$
(\mathcal{F} g)(z)=\alpha_{0} \delta+\frac{1}{|z|^{2}} h\left(e^{i \operatorname{Arg}(z)}\right)
$$

where the smooth function $h$ on the circle is defined by the formula

$$
h\left(e^{i \theta}\right)=\sum_{0 \neq k \in \mathbb{Z}} \frac{|k|}{2 \pi i^{k}} \alpha_{k} e^{i k \theta}
$$

So, $\left(\mathcal{F} g-\alpha_{0} \delta\right)(z)=O\left(|z|^{-2}\right)$. Furthermore, have

$\nabla\left(\frac{h\left(e^{i \operatorname{Arg}(\mathrm{z})}\right)}{|z|^{2}}\right)=h\left(e^{i \operatorname{Arg}(\mathrm{z})}\right) \cdot \nabla\left(\frac{1}{|z|^{2}}\right)+\left.\frac{1}{|z|^{2}} \cdot \frac{d h\left(e^{i \theta}\right)}{d \theta}\right|_{\theta=\operatorname{Arg}(z)} \cdot \nabla(\operatorname{Arg}(z))=O\left(\frac{1}{|z|^{3}}\right)$.

This completes the verification that $\mathcal{F} g-\alpha_{0} \delta$ satisfies condition (2.2).

Theorem 4.4. For every $A=A^{*} \in \mathcal{M}$ with $\operatorname{spec}(A) \subset \mathbb{Z}$ and for every Lipschitz function $f$, we have

$$
\left\|T_{f^{[1]}}^{A, A}(V)\right\|_{1, \infty} \leq c_{a b s}\left\|f^{\prime}\right\|_{\infty}\|V\|_{1}, \quad V \in L_{1}(\mathcal{M}) .
$$

Proof. Fix a smooth homogeneous function $g$ on $\mathbb{R}^{2}$ such that $g\left(e^{i \theta}\right)=\tan (\theta)$ for $\theta \in\left(-\frac{\pi}{4}, \frac{\pi}{4}\right)$ and for $\theta \in\left(\frac{3 \pi}{4}, \frac{5 \pi}{4}\right)$. Without loss of generality, $g$ is mean zero on the circle $\{|z|=1\}$. By Lemma 4.3, $\mathcal{F} g$ satisfies the conditions (2.2). The operator $g(\nabla) \in B\left(L_{2}\left(\mathbb{R}^{2}\right)\right)$ since $g$ is bounded. Recall that $(g(\nabla))(x)=(\mathcal{F} g) * x$. By Theorem 2.1, we have

$$
1 \otimes g(\nabla): L_{1}\left(\mathcal{M} \otimes L_{\infty}\left(\mathbb{R}^{2}\right)\right) \rightarrow L_{1, \infty}\left(\mathcal{M} \otimes L_{\infty}\left(\mathbb{R}^{2}\right)\right)
$$

Consider Schwartz functions $5 \phi_{m}$ on $\mathbb{R}^{2}$ which vanish near 0 , such that $\phi_{m}(t)=1$ for $|t| \in\left(\frac{1}{m}, m\right)$ and such that $\left\|\mathcal{F} \phi_{m}\right\|_{1} \leq c_{a b s}$ for all $m \geq 1$. It follows that

$$
\begin{aligned}
& \left\|1 \otimes\left(g \phi_{m}\right)(\nabla)\right\|_{L_{1} \rightarrow L_{1, \infty}} \leq\|1 \otimes g(\nabla)\|_{L_{1} \rightarrow L_{1, \infty}}\left\|1 \otimes \phi_{m}(\nabla)\right\|_{L_{1} \rightarrow L_{1}} \leq \\
\leq & \|1 \otimes g(\nabla)\|_{L_{1} \rightarrow L_{1, \infty}}\left\|\mathcal{F} \phi_{m}\right\|_{1} \leq c_{a b s}\|1 \otimes g(\nabla)\|_{L_{1} \rightarrow L_{1, \infty}}=c_{a b s}, \quad m \geq 1 .
\end{aligned}
$$

The last equality holds because $g$ is fixed.

\footnotetext{
${ }^{4}$ This can be checked e.g. by substituting $m=\Omega=g_{k}$ into the formula (26) in Theorem II.4.3 in 28 .

${ }^{5}$ Let $\psi$ be a Schwartz function on $\mathbb{R}$ which is 1 on $(-1,1)$ and which is supported on $(-2,2)$. Set $\psi_{m}=\sigma_{m} \psi \cdot\left(1-\sigma_{\frac{1}{m}} \psi\right)$. It follows that

$$
\mathcal{F}\left(\psi_{m}\right)=\mathcal{F}\left(\sigma_{m} \psi\right) * \mathcal{F}\left(1-\sigma_{\frac{1}{m}} \psi\right)=m \sigma_{\frac{1}{m}}(\mathcal{F}(\psi))-m \sigma_{\frac{1}{m}}(\mathcal{F}(\psi)) * \frac{1}{m} \sigma_{m}(\mathcal{F}(\psi)) .
$$
}

Applying Young's inequality, we conclude that

$$
\left\|\mathcal{F}\left(\psi_{m}\right)\right\|_{1} \leq\left\|m \sigma_{\frac{1}{m}}(\mathcal{F}(\psi))\right\|_{1}+\left\|m \sigma_{\frac{1}{m}}(\mathcal{F}(\psi))\right\|_{1}\left\|\frac{1}{m} \sigma_{m}(\mathcal{F}(\psi))\right\|_{1}=\|\mathcal{F}(\psi)\|_{1}+\|\mathcal{F}(\psi)\|_{1}^{2} .
$$

Consider the functions $\phi_{m}=\psi_{3 m}^{\otimes 2}$. By Fubini Theorem, $\sup _{m \geq 1}\left\|\mathcal{F}\left(\phi_{m}\right)\right\|_{1}<\infty$. Clearly, $\psi_{m}=1$ on the set $[-m, m] \backslash\left[-\frac{2}{m}, \frac{2}{m}\right]$. Thus, $\phi_{m}(t)=1$ if $t \in 3 m K$ and $3 m t \notin 2 K$, where $K=[-1,1] \times$ $[-1,1]$. Thus, $\phi_{m}(t)=1$ whenever $|t| \in\left(\frac{1}{m}, m\right)$. 
By assumption, $A=\sum_{j \in \mathbb{Z}} j p_{j}$, where $\left\{p_{j}\right\}_{j \in \mathbb{Z}}$ are pairwise orthogonal projections such that $\sum_{j \in \mathbb{Z}} p_{j}=1$. Since $A$ is bounded, it follows that $p_{j}=0$ for all but finitely many $j \in \mathbb{Z}$. Hence, sums are, in fact, finite. Consider a unitary operator

$$
u=\sum_{j \in \mathbb{Z}} p_{j} \otimes e_{(j, f(j))},
$$

where $e_{(j, f(j))}$ is given in (3.4). Without loss of generality, $\left\|f^{\prime}\right\|_{\infty} \leq 1$. For every $m \geq\|A\|_{\infty}$, we have $|i-j|,|f(i)-f(j)| \leq 2 m$ for every $i, j \in \operatorname{spec}(A)$. Hence,

$\left(g \phi_{m}\right)(i-j, f(i)-f(j))=g(i-j, f(i)-f(j))=\frac{f(i)-f(j)}{i-j}, \quad i, j \in \operatorname{spec}(A), \quad i \neq j$.

It follows from the preceding paragraph and from the equality $\left\|G_{l}^{\otimes 2}\right\|_{1}=1$ that

$$
\left\|\left(1 \otimes\left(g \phi_{m}\right)(\nabla)\right)\left(u\left(V \otimes G_{l}^{\otimes 2}\right) u^{*}\right)\right\|_{1, \infty} \leq c_{a b s}\left\|u\left(V \otimes G_{l}^{\otimes 2}\right) u^{*}\right\|_{1}=c_{a b s}\|V\|_{1} .
$$

It is clear that

$$
\left(1 \otimes\left(g \phi_{m}\right)(\nabla)\right)\left(u\left(V \otimes G_{l}^{\otimes 2}\right) u^{*}\right)=\sum_{i, j} p_{i} V p_{j} \otimes\left(g \phi_{m}(\nabla)\right)\left(G_{l}^{\otimes 2} e_{(i-j, f(i)-f(j))}\right) .
$$

Since there are only finitely many summands, it follows from Lemma 3.3 (as applied to the Schwartz function $g \phi_{m}$ ) that

$$
\left(1 \otimes\left(g \phi_{m}\right)(\nabla)\right)\left(u\left(V \otimes G_{l}^{\otimes 2}\right) u^{*}\right)-\sum_{i \neq j} p_{i} V p_{j} \otimes \frac{f(i)-f(j)}{i-j} G_{l}^{\otimes 2} e_{(i-j, f(i)-f(j))} \rightarrow 0
$$

in $L_{1}\left(\mathcal{M} \otimes L_{\infty}\left(\mathbb{R}^{2}\right)\right)$ as $l \rightarrow \infty$. It is immediate that

$$
\begin{gathered}
\sum_{i \neq j} p_{i} V p_{j} \otimes \frac{f(i)-f(j)}{i-j} G_{l}^{\otimes 2} e_{(i-j, f(i)-f(j))}= \\
=\left(\sum_{k \in \mathbb{Z}} p_{k} \otimes e_{(k, f(k))}\right) \cdot\left(\sum_{i \neq j} p_{i} V p_{j} \otimes \frac{f(i)-f(j)}{i-j} G_{l}^{\otimes 2}\right) \cdot\left(\sum_{l \in \mathbb{Z}} p_{l} \otimes e_{(-l,-f(l))}\right)= \\
\stackrel{(2.5)}{=} u\left(T_{f^{[1]}}^{A, A}(V) \otimes G_{l}^{\otimes 2}\right) u^{*} .
\end{gathered}
$$

Therefore,

$$
\left(1 \otimes\left(g \phi_{m}\right)(\nabla)\right)\left(u\left(V \otimes G_{l}^{\otimes 2}\right) u^{*}\right)-u\left(T_{f^{[1]}}^{A, A}(V) \otimes G_{l}^{\otimes 2}\right) u^{*} \rightarrow 0
$$

in $L_{1}\left(\mathcal{M} \otimes L_{\infty}\left(\mathbb{R}^{2}\right)\right)$ (and, hence, in $\left.L_{1, \infty}\left(\mathcal{M} \otimes L_{\infty}\left(\mathbb{R}^{2}\right)\right)\right)$ as $l \rightarrow \infty$.

Combining (4.2) and (4.3), we arrive at

$$
\limsup _{l \rightarrow \infty}\left\|u\left(T_{f^{[1]}}^{A, A}(V) \otimes G_{l}^{\otimes 2}\right) u^{*}\right\|_{1, \infty} \leq c_{a b s}\|V\|_{1} .
$$

Since $u$ is unitary, it follows that

$$
\limsup _{l \rightarrow \infty}\left\|T_{f^{[1]}}^{A, A}(V) \otimes G_{l}^{\otimes 2}\right\|_{1, \infty} \leq c_{a b s}\|V\|_{1} .
$$

The assertion follows now from Lemma 4.2. 


\section{Proof of the MAIN Results}

In this section we collect the results announced in the abstract and its corollaries. Throughout this section fix a semi-finite von Neumann algebra $\mathcal{M}$ with normal, semi-finite, faithful trace $\tau$.

Lemma 5.1. Let $A=A^{*} \in \mathcal{M}$. If $\left\{\xi_{n}\right\}_{n \geq 0}$ is a uniformly bounded sequence of Borel functions on $\mathbb{R}^{2}$ such that $\xi_{n} \rightarrow \xi$ everywhere, then

$$
T_{\xi_{n}}^{A, A}(V) \rightarrow T_{\xi}^{A, A}(V), \quad V \in L_{2}(\mathcal{M})
$$

in $L_{2}(\mathcal{M})$ as $n \rightarrow \infty$.

Proof. Let $\nu$ be a projection valued measure on $\mathbb{R}^{2}$ considered in Subsection 2.4 (see (2.4)). Let $\gamma: \mathbb{R} \rightarrow \mathbb{R}^{2}$ be a Borel measurable bijection. Clearly, $\nu \circ \gamma$ is a projection valued measure on $\mathbb{R}$. Hence, there exists a self-adjoint operator $B$ acting on the Hilbert space $L_{2}(\mathcal{M})$ such that $E_{B}=\nu \circ \gamma$.

Set $\eta_{n}=\xi_{n} \circ \gamma$ and $\eta=\xi \circ \gamma$. We have $\eta_{n} \rightarrow \eta$ everywhere on $\mathbb{R}$. Thus,

$$
\begin{gathered}
T_{\xi_{n}}^{A, A}=\int_{\mathbb{R}^{2}} \xi_{n} d \nu=\int_{\mathbb{R}} \eta_{n}(\lambda) d E_{B}(\lambda)=\eta_{n}(B) \rightarrow \eta(B)= \\
=\int_{\mathbb{R}} \eta(\lambda) d E_{B}(\lambda)=\int_{\mathbb{R}^{2}} \xi d \nu=T_{\xi}^{A, A} .
\end{gathered}
$$

Here, the convergence is understood with respect to the strong operator topology on the space $B\left(L_{2}(\mathcal{M})\right.$ ). In particular, (5.1) follows.

Proof of Theorem 1.2. Step 1. Let $A$ is bounded. For every $n \geq 1$, set

$$
\begin{gathered}
A_{n} \stackrel{\text { def }}{=} \sum_{k \in \mathbb{Z}} \frac{k}{n} E_{A}\left(\left[\frac{k}{n}, \frac{k+1}{n}\right)\right), \\
\xi_{n}(t, s)=f^{[1]}\left(\frac{k}{n}, \frac{l}{n}\right), \quad t \in\left[\frac{k}{n}, \frac{k+1}{n}\right), s \in\left[\frac{l}{n}, \frac{l+1}{n}\right) .
\end{gathered}
$$

It is immediate that (see e.g. Lemma 8 in [25] for much stronger assertion)

$$
T_{\xi_{n}}^{A, A}(V)=T_{f^{[1]}}^{A_{n}, A_{n}}(V)=T_{\left(n \sigma_{n} f\right)^{[1]}}^{n A_{n}, n A_{n}}(V) .
$$

It follows from Theorem 4.4 that

$$
\left\|T_{\xi_{n}}^{A, A}(V)\right\|_{1, \infty} \leq c_{a b s}\left\|\left(n \sigma_{n} f\right)^{\prime}\right\|_{\infty}\|V\|_{1}=c_{a b s}\left\|f^{\prime}\right\|_{\infty}\|V\|_{1} .
$$

Note that $\xi_{n} \rightarrow f^{[1]}$ everywhere. It follows from Lemma 5.1 that

$$
T_{\xi_{n}}^{A, A}(V) \rightarrow T_{f^{[1]}}(V), \quad V \in L_{2}(\mathcal{M})
$$

in $L_{2}(\mathcal{M})$ (and, hence, in measure - see e.g [20]) as $n \rightarrow \infty$. Since the quasi-norm in $L_{1, \infty}(\mathcal{M})$ is a Fatou quasi-norm, it follows that

$$
\left\|T_{f^{[1]}}^{A, A}(V)\right\|_{1, \infty} \leq c_{a b s}\left\|f^{\prime}\right\|_{\infty}\|V\|_{1}, \quad V \in\left(L_{1} \cap L_{2}\right)(\mathcal{M}) .
$$

Step 2. Let now $A$ be an arbitrary operator affiliated with $\mathcal{M}$. Set $A_{n}=$ $A E_{A}([-n, n])$. By Step 1, we have

$$
\left\|T_{f^{[1]}}^{A_{n}, A_{n}}(V)\right\|_{1, \infty} \leq c_{a b s}\left\|f^{\prime}\right\|_{\infty}\|V\|_{1}
$$

It follows immediately from the definition of the double operator integral that

$$
T_{f^{[1]}}^{A_{n}, A_{n}}(V)=E_{A}([-n, n]) \cdot T_{f^{[1]}}^{A, A}(V) \cdot E_{A}([-n, n]) \rightarrow T_{f^{[1]}}^{A, A}(V)
$$


in $L_{2}(\mathcal{M})$ (and, hence, in measure) as $n \rightarrow \infty$. Since the quasi-norm in $L_{1, \infty}(\mathcal{M})$ is a Fatou quasi-norm, the assertion follows.

The following lemma is ideologically similar to Theorem 7.4 in [20].

Lemma 5.2. If $A, B \in \mathcal{M}$ are such that $[A, B] \in L_{2}(\mathcal{M})$, then, for every Lipschitz function $f$, we have

$$
T_{f^{[1]}}^{A, A}([A, B])=[f(A), B] .
$$

Proof. By definition of double operator integral given in Subsection 2.4 we have

$$
T_{\xi_{1}}^{A, A} T_{\xi_{2}}^{A, A}=T_{\xi_{1} \xi_{2}}^{A, A} .
$$

Let $\xi_{1}=f^{[1]}$ and let $\xi_{2}(\lambda, \mu)=\lambda-\mu$ when $|\lambda|,|\mu| \leq\|A\|_{\infty} \cdot \xi_{2}(\lambda, \mu)=0$ when $|\lambda|>\|A\|_{\infty}$ or $|\mu| \leq\|A\|_{\infty}$.

If $p$ is a $\tau$-finite projection, then $p B \in L_{2}(\mathcal{M})$ and

$$
T_{\xi_{1} \xi_{2}}^{A, A}(p B)=f(A) p B-p B f(A), \quad T_{\xi_{2}}^{A, A}(p B)=A p B-p B A,
$$

Applying (5.2) to the operator $p B \in L_{2}(\mathcal{M})$, we obtain

$$
T_{f^{[1]}}^{A, A}(A p B-p B A)=f(A) p B-p B f(A) .
$$

Applying Proposition 6.6 in [20] to the operator $n A$, we construct a sequence $\left\{p_{n, k}\right\}_{k \geq 0}$ of $\tau$-finite projections such that $p_{n, k} \uparrow 1$ as $k \rightarrow \infty$ and such that $\left\|\left[n A, p_{n, k}\right]\right\|_{2} \leq 1$. Let $\left\{\eta_{m}\right\}_{m \geq 0}$ be an orthonormal basis in $L_{2}(\mathcal{M})$. Fix $k_{n}$ so large that

$$
\left\|\left(1-p_{n, k_{n}}\right) \eta_{m}\right\|_{2} \leq \frac{1}{n}, \quad 0 \leq m<n .
$$

Set $q_{n}=p_{n, k_{n}}$. It follows from (5.4) that $q_{n} \rightarrow 1$ in the strong operator topology (in the left regular representation of $\mathcal{M}$ ). Clearly, $\left[A, q_{n}\right] \rightarrow 0$ in $L_{2}(\mathcal{M})$.

By construction,

$$
A q_{n} B-q_{n} B A=\left[A, q_{n}\right] B+q_{n}[A, B] \rightarrow[A, B], \quad n \rightarrow \infty,
$$

in $L_{2}(\mathcal{M})$. Since $T_{f^{[1]}}^{A, A}$ is bounded, it follows that

$$
f(A) q_{n} B-q_{n} B f(A) \stackrel{\left(\frac{5.3)}{=}\right.}{=} T_{f^{[1]}}^{A, A}\left(A q_{n} B-q_{n} B A\right) \rightarrow T_{f^{[1]}}^{A, A}(A B-B A), \quad n \rightarrow \infty,
$$

in $L_{2}(\mathcal{M})$. On the other hand,

$$
f(A) q_{n} B-q_{n} B f(A) \rightarrow[f(A), B], \quad n \rightarrow \infty,
$$

in the strong operator topology (in the left regular representation of $\mathcal{M}$ ). This concludes the proof.

Theorem 5.3. For all self-adjoint operators $A, B \in \mathcal{M}$ such that $[A, B] \in L_{1}(\mathcal{M})$ and for every Lipschitz function $f$, we have

$$
\|[f(A), B]\|_{1, \infty} \leq c_{a b s}\left\|f^{\prime}\right\|_{\infty} \cdot\|[A, B]\|_{1} .
$$

For all self-adjoint operators $X, Y \in \mathcal{M}$ such that $X-Y \in L_{1}(\mathcal{M})$ and for every Lipschitz function $f$, we have

$$
\|f(X)-f(Y)\|_{1, \infty} \leq c_{a b s}\left\|f^{\prime}\right\|_{\infty}\|X-Y\|_{1} .
$$


Proof. By assumption, $[A, B] \in\left(L_{1} \cap L_{2}\right)(\mathcal{M})$. The first assertion follows by combining Lemma 5.2 and Theorem 1.2 Applying the first assertion to the operators

$$
A=\left(\begin{array}{cc}
X & 0 \\
0 & Y
\end{array}\right), \quad B=\left(\begin{array}{ll}
0 & 1 \\
1 & 0
\end{array}\right),
$$

we obtain the second assertion.

\section{REFERENCES}

[1] M. S. Birman and M. Z. Solomyak, Double Stieltjes operator integrals (Russian), Probl. Math. Phys., Izdat. Leningrad. Univ., Leningrad, (1966) 33-67. English translation in: Topics in Mathematical Physics, Vol. 1 (1967), Consultants Bureau Plenum Publishing Corporation, New York, 25-54.

[2] Birman M., Solomyak M. Spectral theory of selfadjoint operators in Hilbert space. Mathematics and its Applications (Soviet Series). D. Reidel Publishing Co., Dordrecht, 1987.

[3] Caspers M., Montgomery-Smith S., Potapov D., Sukochev F. The best constants for operator Lipschitz functions on Schatten classes. J. Funct. Anal. 267 (2014), no. 10, 3557-3579.

[4] Caspers M., Potapov D., Sukochev F., Zanin D. Weak type estimates for the absolute value mapping. J. Operator Theory, 73 (2015), no. 2, 101-124.

[5] Davies E. Lipschitz continuity of functions of operators in the Schatten classes. J. Lond. Math. Soc. 37 (1988) 148-157.

[6] de Leeuw, K. On $L_{p}$ multipliers. Ann. of Math. (2) 81 (1965) 364-379.

[7] Dodds P., Dodds T., de Pagter B., Sukochev F. Lipschitz continuity of the absolute value and Riesz projections in symmetric operator spaces. J. Funct. Anal. 148 (1997), 28-69.

[8] Dodds P., Dodds T., de Pagter B., Sukochev F. Lipschitz continuity of the absolute value in preduals of semifinite factors. Integral Equations Operator Theory 34 (1999), 28-44.

[9] Farforovskaya Y. An estimate of the nearness of the spectral decompositions of self-adjoint operators in the Kantorovich-Rubinstein metric. Vestnik Leningrad. Univ. 22 (1967), no. 19, $155-156$.

[10] Farforovskaya Y. The connection of the Kantorovich-Rubinstein metric for spectral resolutions of selfadjoint operators with functions of operators. Vestnik Leningrad. Univ. 23 (1968), no. 19, 94-97.

[11] Farforovskaya Y. An example of a Lipschitz function of self-adjoint operators with nonnuclear difference under a nuclear perturbation. Zap. Nauchn. Sem. Leningrad. Otdel. Mat. Inst. Steklov. (LOMI) 30 (1972), 146-153.

[12] Grafakos L. Classical Fourier analysis. Third edition. Graduate Texts in Mathematics, 249. Springer, New York, 2014.

[13] Halmos P., von Neumann J. Operator methods in classical mechanics. II. Ann. of Math. (2) 43, (1942). 332-350.

[14] Kalton N., Sukochev F. Symmetric norms and spaces of operators. J. Reine Angew. Math. 621 (2008), 81-121.

[15] Kato T. Continuity of the map $S \mapsto|S|$ for linear operators. Proc. Japan Acad. 49 (1973) 157-160.

[16] Kosaki H. Unitarily invariant norms under which the map $A \mapsto|A|$ is continuous. Publ. Res. Inst. Math. Sci. 28 (1992), 299-313.

[17] Krein M. Some new studies in the theory of perturbations of self-adjoint operators. First Math. Summer School, Part I (Russian), Izdat. "Naukova Dumka", Kiev, 1964, pp. 103-187.

[18] Lord S., Sukochev F., Zanin D. Singular traces. Theory and applications. De Gruyter Studies in Mathematics, 46. De Gruyter, Berlin, 2013.

[19] Nazarov F., Peller V. Lipschitz functions of perturbed operators. C. R. Math. Acad. Sci. Paris 347 (2009), 857-862.

[20] de Pagter B., Witvliet H., Sukochev F. Double operator integrals. J. Funct. Anal. 192 (2002), no. $1,52-111$.

[21] Parcet J. Pseudo-localization of singular integrals and noncommutative Calderón-Zygmund theory. J. Funct. Anal. 256 (2009), 509-593.

[22] Peller V. Hankel operators in the theory of perturbations of unitary and selfadjoint operators. Funktsional. Anal. i Prilozhen. 19 (1985), no. 2, 37-51, 96. 
[23] Potapov D., Sukochev F. Lipschitz and commutator estimates in symmetric operator spaces. J. Operator Theory 59 (2008) 211-234.

[24] Potapov D., Sukochev F. Operator-Lipschitz functions in Schatten-von Neumann classes. Acta Math. 207 (2011), no. 2, 375-389.

[25] Potapov D., Sukochev F. Unbounded Fredholm modules and double operator integrals. J. Reine Angew. Math. 626 (2009), 159-185.

[26] Randrianantoanina N. A weak type inequality for non-commutative martingales and applications. Proc. London Math. Soc. (3) 91 (2005), no. 2, 509-542.

[27] de la Salle M. A shorter proof of a result by Potapov and Sukochev on Lipschitz functions on $S_{p}$. arXiv:0905.1055

[28] Stein E. Singular integrals and differentiability properties of functions. Princeton Mathematical Series, No. 30 Princeton University Press, Princeton, N.J.

[29] Sukochev F. On a conjecture of A. Bikchentaev. Proc. Sympos. Pure Math., 87, Amer. Math. Soc., Providence, R.I., 2013.

[30] Sukochev F. Completeness of quasi-normed symmetric operator spaces. Indag. Math. (N.S.) 25 (2014), no. 2, 376-388.

M. Caspers, Fachbereich Mathematik und Informatik der Universität Münster, EinSteinstrasse 62, 48149 MÜnster, Germany

E-mail address: martijn.caspers@uni-muenster.de

D. Potapov, F. Sukochev, D. Zanin, School of Mathematics and Statistics, UnSW, Kensington 2052, NSW, Australia

E-mail address: d.potapov@unsw.edu.au

E-mail address: f.sukochev@unsw.edu.au

E-mail address: d.zanin@unsw.edu.au 